\title{
Assessing the twinning model in the Rwandan Human Resources for Health Program: goal setting, satisfaction and perceived skill transfer
}

Esperance Ndenga ${ }^{1}$, Glorieuse Uwizeye ${ }^{1 *}$, Dana R. Thomson ${ }^{2,3}$, Eric Uwitonze ${ }^{1}$, Joel Mubiligi ${ }^{1}$, Bethany L. Hedt-Gauthier ${ }^{2,3}$, Michael Wilkes ${ }^{2,3}$ and Agnes Binagwaho $3,4,5$

\begin{abstract}
Background: Because of the shortage of health professionals, particularly in specialty areas, Rwanda initiated the Human Resources for Health (HRH) Program. In this program, faculty from United States teaching institutions (USF) "twin" with Rwandan Faculty (RF) to transfer skills. This paper assesses the twinning model, exploring USF and RF goal setting, satisfaction and perceptions of the effectiveness of skill transfer within the twinning model.

Methods: All USF and RF in the HRH Program from August 2012-May 2014 were invited to participate. An 85-item questionnaire for USF and 71-item questionnaire for RF were administered via Survey Monkey in April and May 2014. Associations among primary outcomes were assessed and factors related with outcomes were modeled using logistic regression.

Results: Most RF and USF reported setting goals with their twin (89\% and $71 \%$, respectively). Half of RF (52\%) reported effective skill transfer compared to $10 \%$ of USF. Only $38 \%$ of RF and $28 \%$ of USF reported being very satisfied with the twinning model. There was significant overlap in the three operational outcomes. For RF, the following factors were associated with outcomes: for effective skill transfer, being able to communicate in a common language and working at a nursing site outside of Kigali; and for satisfaction, 7+ years of professional experience and being part of a male RF-female USF twin pair. For USF, the following factors were associated with outcomes: for setting goals, prior teaching experience; and for satisfaction, experience in low resource settings for one month or less and feeling that HRH promotes a culture of respect.

Conclusions: Twinning is the cornerstone of the HRH Program in Rwanda. These findings helped the HRH team identify key areas to improve the twinning experience including better recruitment and orientation of USF and RF, consideration of additional factors during the twinning process, provide language training support, facilitate joint twin activities and cross-cultural training and improve the site leadership buy-in and support of the program. These results can inform other programs using twinning to develop skills in the health workforce.
\end{abstract}

Keywords: Africa, Human resources, Health care workers, Training, Twinning, Mentorship

\footnotetext{
* Correspondence: uglorieuse@gmail.com

${ }^{1}$ Human Resources for Health, Ministry of Health, P.O. Box 84, Kigali, Rwanda

Full list of author information is available at the end of the article
} 


\section{Background}

With the established link between positive health outcomes and a sufficient number of well-qualified heath workers [1-3], developing a skilled workforce is a global priority [2]. In 2006, the World Health Organization (WHO) estimated that 4.3 million health professionals were needed globally to provide basic health services [2]. Developed countries face challenges in terms of inequitable geographical and skills distribution $[4,5]$. In addition to these pressures, low and middle income countries are impacted by the migration of health workers as thousands of doctors, nurses and midwives emigrate for better work and living conditions after their countries have invested heavily in their education [6-10].

Africa, particularly sub-Saharan Africa, is highly affected by its limited health workforce. While Africa bears $24 \%$ of the global disease burden, it employs only $3 \%$ of healthcare workers worldwide [2]. With a staffing ratio far below the recommended 2.3 healthcare professionals per 1000 population needed to achieve the Millennium Development Goals (MDGs) [11], questions were raised as to whether countries in Africa would be able to meet the health-related MDGs $[12,13]$. Indeed, while there was progress in all MDGs in sub-Saharan Africa, none of the targets were met for the region by the 2015 end-line evaluation [14].

Sub-Saharan Africa faces two critical issues: 1) the quantity/distribution of healthcare providers and 2) the skills of those providers, particularly in specialty areas. Many countries have deployed government and donor funds for long-term programs to address these challenges. In 2005, Malawi secured USD\$272 million for a five-year program to train and retain health professionals [15]. In Tanzania, professional health education has evolved from one medical school in 1963 to eight universities in 2009 each containing various health-professional training programs, thus increasing the annual intake of medical students by 15 fold in the last two decades [16].

Similar to other African countries, Rwanda has a persistent shortage of qualified health professionals but the issue was heightened by the 1994 Genocide against the Tutsi where a large number of health workers were killed and others fled the country [17]. In the aftermath of the Genocide, Rwanda has invested in reconstructing the health infrastructure and workforce. As a result, the country has made dramatic progress towards achieving the health-related MDGs [18] and life expectancy has increased from 48 years in 1990 to 65 years in 2012 [19]. The only medical school in the country has increased its graduation rate from an annual average of 15 graduates in its first 25 years to five times as many in 2004-2010 [20]. Until 1998, physician specialists, in areas such as surgery, internal medicine, pediatrics, obstetrics and gynecology, were educated abroad. In 1998, the University of Rwanda created postgraduate programs to train in some of these specialty areas in country. Further, to prevent "brain drain" of health care professionals to high paying developed countries, Rwanda has implemented several strategies, including providing work contracts for health professionals in training, appointing new graduates in a timely manner and implementing performance-based financing [21].

Even with these changes, Rwanda still struggles to increase the number of healthcare professionals (doctor, nurses and midwives) to the minimum recommended by the WHO and to train health care specialists. In 2012, the Rwandan Ministry of Health, with support of the Centers for Diseases Control and Prevention and The Global Fund to Fight AIDS, Tuberculosis and Malaria, initiated the innovative 7-year Human Resources for Health (HRH) Program [21, 22]. The overall aim of $\mathrm{HRH}$ Program is to increase the quality of health care in Rwanda through the training of sufficient number of highly qualified health professionals capable of providing world-class care. Instead of sending Rwandan professionals to high income countries to receive specialty and subspecialty training, the HRH Program hires high caliber faculty from 23 United States teaching institutions (USI) in the fields of nursing, midwifery, medicine, dentistry, health management and public health to work in Rwanda [22]. These USI faculty (USF) are "twinned" incountry with Rwandan faculty (RF) at university and clinical teaching sites. The goal of the twinning process is to transfer skills to the RF, who in turn will continue to educate future health professionals and mentor new $\mathrm{RF}$ in a manner that is sustainable and cost effective.

Evidence of impact of international partnerships is limited but critical for maximizing partnership effectiveness [23]. While randomized controlled trials for such evaluations are generally not feasible, institutions engaged in partnerships are encouraged to evaluate components of the partnership and share lessons learned [24]. This paper uses RF and USF perceptions to evaluate the twinning experience, specifically in the areas of goal setting, skills transfer and participant satisfaction. These three areas were chosen because they are on the pathway to successful HRH twinning and identifying factors related to these areas can generate recommendations to improve the HRH Program and clinical training in other low-resource countries.

\section{Methods}

The Human Resources for Health Program in Rwanda

Since its inception in 2012, over 313 USF and 210 RF have been engaged in the HRH Program. The HRH Program targets four disciplines for RF development: medicine, nursing/midwifery, oral health and healthcare management. Each year, the University of Rwanda College of Medicine 
and Health Sciences (UR-CMHS) selects priorities in each discipline for specialized skills development. RF are selected at the department level at each teaching site with consideration of department goals, personal goals and skill of potential Rwandan twins.

Partner institutions in the United States screen potential USF candidates and submit these candidates for University of Rwanda consideration through the HRH team. RF and USF already based in Rwanda then advise on these applications and recommend candidates, based on their suitability for a RF twin, to subcommittees for approval. Approved USF are contracted by the respective USI for periods of time that are typically one year, except for physician subspecialists who rotate for a minimum of 8 weeks.

Orientation is provided to USF by their home institutions prior to their departure for Rwanda. The HRH Program also organizes a pre-departure orientation phone call that focuses on programmatic and logistic matters. Once in Rwanda, HRH staff in the $\mathrm{MOH}$ welcome USF and introduce them to the country, and their placements, head of department and twin. Further, the $\mathrm{HRH}$ staff in the $\mathrm{MOH}$ organize a pre-orientation meeting for RF and site leadership to help them prepare to receive the USF. In August, usually within one month of the USF arrival, an annual orientation event is organized by the MOH for both USF and RF. The orientation is expected to continue at the site level by site leadership as needed. At the beginning of each year, the twin-pair is expected to set goals to be achieved during their time together; however, they are given autonomy in how they achieve these goals. HRH staff in $\mathrm{MOH}$ and sites leadership provide the needed support for twins to implement their goals and ensure the follow-up on progress made throughout the twinning process.

\section{Study design}

All USF and RF engaged in the HRH Program from August 2012 to May 2014 were invited by email to respond to an online questionnaire about their perspectives and attitudes of the twinning process, program management and possible cultural and interaction challenges. The 85 -item questionnaire for USF and the 71-item questionnaire for RF, administered via Survey Monkey, were identical except the USF questionnaire included additional questions about preparation and adjustment to Rwanda. The RF could opt to take the survey in either French or English, with the French version translated and back translated to English to ensure consistency in meaning between languages. Both English versions of the questionnaire were piloted among health professionals not engaged with twinning program faculty to test the flow of the question and time for completion. Data were collected in April and May 2014. Participation in the survey was optional. To increase participation, reminders were sent by the $\mathrm{HRH}$ staff in the $\mathrm{MOH}$ to all eligible participants.

\section{Analysis}

In the study, three operational outcomes were evaluated based on HRH Program priorities: whether goals had been set, perceived effectiveness of skill transfer from USF-to-RF and satisfaction with the twinning process. A fourth outcome, time spent with twin, was originally considered as a predictor but was reconsidered as an outcome because it likely happens in tandem with goal setting skills transfer and satisfaction. It was not modeled explicitly in this paper, but was considered with other outcomes in the final models.

Twenty-five potential predictors were identified in a conceptual framework, grouped in the following factors (Fig. 1):

- USF recruitment or RF appointments: for example, time since training, previous teaching experience, experience working overseas or with Westerners before entering in the HRH Program;

- The twinning of USF and RF: for example, gender differences, age differences, number of twins, shared languages;

- Factors addressable during orientation or service: for example, understanding of HRH Program goals, understand twinning model, cultural differences; and

- Factors related to program management: for example, perception of support from the site leadership, feel HRH Program promotes culture of respect.

We used univariate logistic regression to test independent associations between each predictor and outcome $(p<0.1)$. Potential significant factors were considered for multivariate logistic regression models that were built using backward stepwise regression, stopping when remaining variables were associated with the outcome at the alpha $=$ 0.1 significance level. The three primary outcomes were modeled independently and separate models were built for USF and RF, since some variables differed between the two types of faculty. Data were cleaned and analyzed in Stata version 13 (College Station, TX: StataCorp LP). We only present direction of association for statistically significant covariates; however, detailed odds ratios and $p$-values are available in Additional file 1.

\section{Ethics statement}

Both the RF and USF surveys were reviewed and approved by HRH Program funders as part of the monitoring and evaluation plan. Since the data was collected for program purposes, the study received a waiver from the Rwandan National Ethical Committee. Participation 

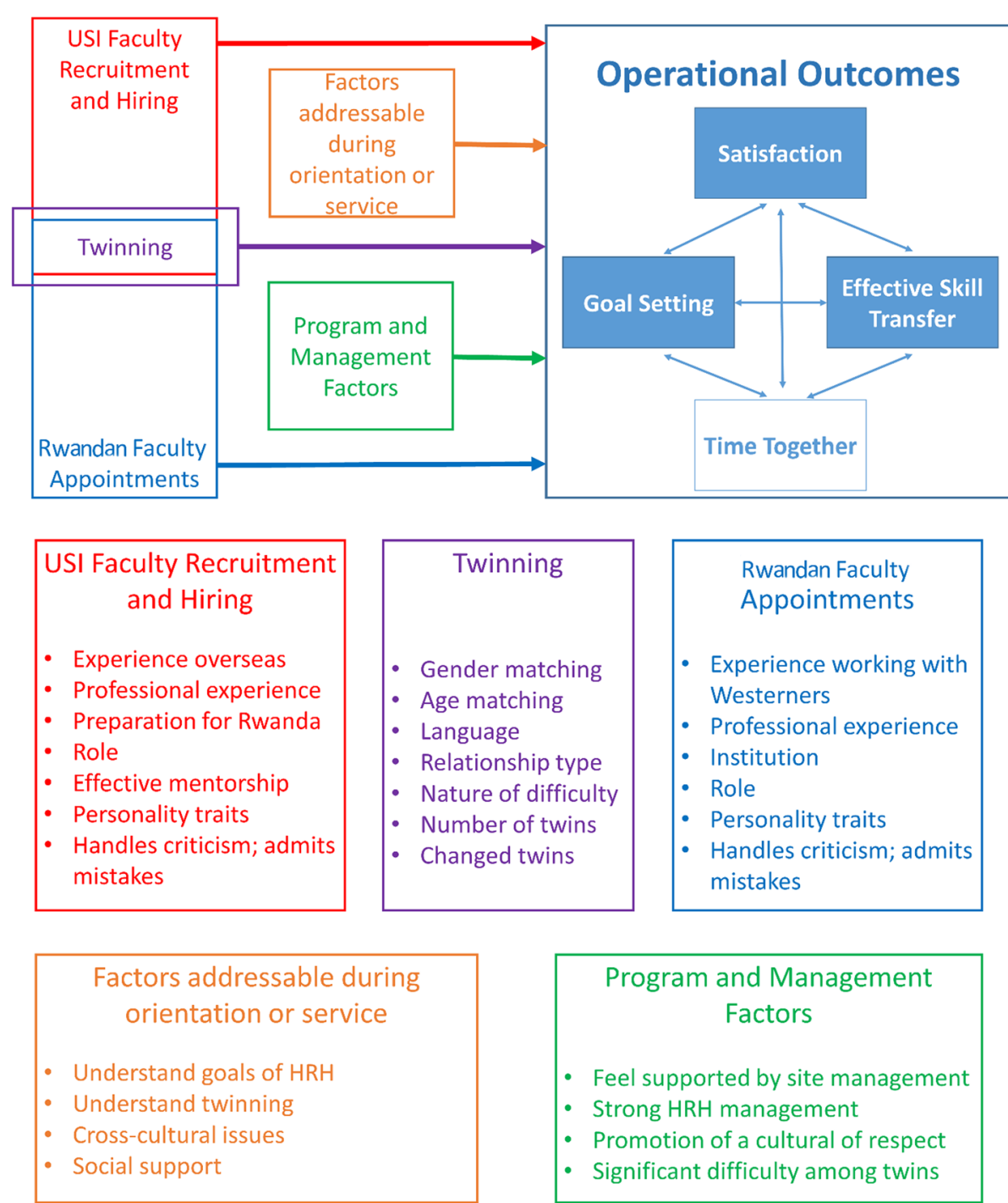

Fig. 1 Conceptual framework of operational outcomes in the Rwandan Human Resources for Health twinning assessment

in the survey was optional and no personal identifying information was collected.

\section{Results}

The response rate among RF was $49 \%(n=71 / 145)$ and from USF $93 \%(n=107 / 112)$. Among USF, 55 (49.1\%) were physicians, 36 (32.1 \%) were nurses/ midwives and $7(6.3 \%)$ were health managers (Table 1). For RF, 14 (19.7 \%) were physicians, 32 $(45.1 \%)$ were nurses/midwives and $5(7.0 \%)$ were health managers. Seventy-three $(65.2 \%)$ of USF had 7+ years working experience post-training compared to 13 $(18.3 \%)$ of RF. Eighty percent $(n=57)$ of RF had a moderate amount of experience working with people from Western cultures before engagement in the HRH Program. Nearly half of the USF $(n=51,46 \%)$ had never worked longer than a month at a time in low-resource countries. For the three primary outcomes, there was significant overlap in prevalence of goal setting, skill transfer and feeling satisfied with the twinning experience overall for both Rwandan and USF (Fig. 2). Results for these three operational outcomes are presented below.

\section{Goal setting}

Among those who responded to questions about goal setting, $89 \%$ of RF and $71 \%$ of USF set goals (Table 1 ). Because none of the tested covariates were associated with goal setting among RF during bivariate analysis, a multivariate model was not built (Table 2). Among USF, a number of hiring and appointment factors (significant prior teaching experience, spent less than a month in lowresource countries at a time prior to engagement in $\mathrm{HRH}$ Program, feel valued by twin, twin handles criticism well), twinning factors (similar ages, excellent communication in 
Table 1 Summary of covariates by faculty type

\begin{tabular}{|c|c|c|c|c|}
\hline & \multicolumn{2}{|c|}{ Rwandan Faculty } & \multicolumn{2}{|c|}{ USI Faculty } \\
\hline & Number & Percent & Number & Percent \\
\hline \multicolumn{5}{|l|}{ OUTCOMES } \\
\hline \multicolumn{5}{|l|}{ Set joint goals with twin } \\
\hline No & 6 & 8.5 & 26 & 23.2 \\
\hline Yes & 47 & 66.2 & 65 & 58.0 \\
\hline Missing & 18 & 25.4 & 21 & 18.8 \\
\hline \multicolumn{5}{|l|}{ Very effective skill transfer (USF-to-RF) } \\
\hline No & 23 & 32.4 & 92 & 82.1 \\
\hline Yes & 25 & 35.2 & 9 & 8.0 \\
\hline Missing & 23 & 32.4 & 11 & 9.8 \\
\hline \multicolumn{5}{|l|}{ Very satisfied with twinning experience overall } \\
\hline No & 33 & 46.5 & 65 & 58.0 \\
\hline Yes & 20 & 28.2 & 26 & 23.2 \\
\hline Missing & 18 & 25.4 & 21 & 18.8 \\
\hline \multicolumn{5}{|l|}{ Number of hours per week spent working with twin } \\
\hline $0-9$ hours & 22 & 31.0 & 42 & 37.5 \\
\hline 10-19 hours & 12 & 16.9 & 17 & 15.2 \\
\hline $20+$ hours & 19 & 26.8 & 32 & 28.6 \\
\hline Missing & 18 & 25.4 & 21 & 18.8 \\
\hline \multicolumn{5}{|l|}{ HIRING AND APPOINTMENTS } \\
\hline \multicolumn{5}{|l|}{ Discipline } \\
\hline Nurse/midwife & 32 & 45.1 & 36 & 32.1 \\
\hline Physician & 14 & 19.7 & 55 & 49.1 \\
\hline Health manager & 5 & 7.0 & 7 & 6.3 \\
\hline Lecturer, academic & 20 & 28.2 & 11 & 9.8 \\
\hline Missing & 0 & 0.0 & 3 & 2.7 \\
\hline \multicolumn{5}{|l|}{ Primary work site } \\
\hline CHUB, CHUK, KFH, RMH, Muhima & 10 & 14.1 & 18 & 16.1 \\
\hline CMHS, multiple & 34 & 47.9 & 80 & 71.4 \\
\hline Nursing outside Kigali & 27 & 38.0 & 14 & 12.5 \\
\hline \multicolumn{5}{|l|}{ Years since training } \\
\hline $7+$ years & 13 & 18.3 & 73 & 65.2 \\
\hline $4-6$ years & 18 & 25.4 & 17 & 15.2 \\
\hline $1-3$ years & 21 & 29.6 & 14 & 12.5 \\
\hline Completed prior to $\mathrm{HRH}$, not yet completed & 19 & 26.8 & 8 & 7.1 \\
\hline \multicolumn{5}{|l|}{ Previous teaching experience } \\
\hline Moderate-little teaching & 48 & 67.6 & 64 & 57.1 \\
\hline Significant teaching & 23 & 32.4 & 48 & 42.9 \\
\hline \multicolumn{5}{|c|}{ Time spent in resource limited countries before HRH } \\
\hline Short trips $<1$ month, none & & & 51 & 45.5 \\
\hline Medium trips $<6$ months & & & 17 & 15.2 \\
\hline Long trips $>6$ months & & & 44 & 39.3 \\
\hline
\end{tabular}


Table 1 Summary of covariates by faculty type (Continued)

\begin{tabular}{|c|c|c|c|c|}
\hline \multicolumn{5}{|l|}{ Applied talent and expertise } \\
\hline \multicolumn{2}{|l|}{ Do not agree } & & 74 & 66.1 \\
\hline \multicolumn{2}{|l|}{ Agree/strongly agree } & & 28 & 25.0 \\
\hline \multicolumn{2}{|l|}{ Missing } & & 10 & 8.9 \\
\hline \multicolumn{5}{|l|}{ Preparation for work in Rwanda } \\
\hline \multicolumn{2}{|l|}{ Well prepared } & & 20 & 17.9 \\
\hline \multicolumn{2}{|l|}{ Moderately prepared } & & 50 & 44.6 \\
\hline \multicolumn{2}{|l|}{ Poorly prepared } & & 29 & 25.9 \\
\hline \multicolumn{2}{|l|}{ Missing } & & 13 & 11.6 \\
\hline \multicolumn{5}{|c|}{ Experience working with people from Western culture before HRH } \\
\hline A lot & 22 & 31.0 & & \\
\hline Moderate & 35 & 49.3 & & \\
\hline None or minimal & 14 & 19.7 & & \\
\hline \multicolumn{5}{|c|}{$\begin{array}{l}\text { Twin values ALL of the following: my expertise, my opinion, our department } \\
\text { hierarchy, my professional interests or goals }\end{array}$} \\
\hline No & 45 & 63.4 & 58 & 51.8 \\
\hline Yes & 2 & 2.8 & 14 & 12.5 \\
\hline Missing & 24 & 33.8 & 40 & 35.7 \\
\hline \multicolumn{5}{|c|}{$\begin{array}{l}\text { Twin shows ANY of the following: withholds information, acts arrogantly, } \\
\text { takes credit for others work, strives for power over others }\end{array}$} \\
\hline No & 28 & 39.4 & 24 & 21.4 \\
\hline Yes & 32 & 45.1 & 80 & 71.4 \\
\hline Missing & 11 & 15.5 & 8 & 7.1 \\
\hline \multicolumn{5}{|c|}{$\begin{array}{l}\text { USI faculty is providing adequate mentorship in at least one of these } \\
\text { roles: educator, clinician, researcher, administrator }\end{array}$} \\
\hline No & 30 & 42.3 & 82 & 73.2 \\
\hline Yes & 23 & 32.4 & 9 & 8.0 \\
\hline Missing & 18 & 25.4 & 21 & 18.8 \\
\hline \multicolumn{5}{|c|}{ Twin handles criticism and admits mistakes quite well or extremely well } \\
\hline Yes & 7 & 9.9 & 10 & 8.9 \\
\hline No & 5 & 7.0 & 19 & 17.0 \\
\hline Missing & 59 & 83.1 & 83 & 74.1 \\
\hline TWINNING & & & & \\
\hline Number of twins ever had & & & & \\
\hline 1 & 34 & 47.9 & 52 & 46.4 \\
\hline 2 & 18 & 25.4 & 29 & 25.9 \\
\hline $3+$ & 12 & 16.9 & 31 & 27.7 \\
\hline Missing & 7 & 9.9 & 0 & 0.0 \\
\hline Changed twins & & & & \\
\hline No change & 37 & 52.1 & 54 & 48.2 \\
\hline Changed one or more times, no longer twinned & 23 & 32.4 & 40 & 35.7 \\
\hline Missing & 11 & 15.5 & 18 & 16.1 \\
\hline
\end{tabular}


Table 1 Summary of covariates by faculty type (Continued)

\begin{tabular}{|c|c|c|c|c|}
\hline \multicolumn{5}{|l|}{ Gender differences } \\
\hline Same gender & 36 & 50.7 & 57 & 50.9 \\
\hline USF female, RF male & 16 & 22.5 & 27 & 24.1 \\
\hline USF male, RF female & 1 & 1.4 & 7 & 6.3 \\
\hline Missing & 18 & 25.4 & 21 & 18.8 \\
\hline \multicolumn{5}{|l|}{ Age differences } \\
\hline$<5$ year difference & 22 & 31.0 & 32 & 28.6 \\
\hline 5-10 year difference & 8 & 11.3 & 25 & 22.3 \\
\hline USF $>10$ years older & 15 & 21.1 & 28 & 25.0 \\
\hline RF $>10$ years older & 8 & 11.3 & 6 & 5.4 \\
\hline Missing & 18 & 25.4 & 21 & 18.8 \\
\hline \multicolumn{5}{|c|}{ Ability to communicate in English, French, or Kinyarwanda } \\
\hline Excellent in at least one & 28 & 39.4 & 52 & 46.4 \\
\hline Moderate, fair, or poor in all & 21 & 29.6 & 39 & 34.8 \\
\hline Missing & 22 & 31.0 & 21 & 18.8 \\
\hline \multicolumn{5}{|l|}{ Relationship with twin } \\
\hline Profession and social, other & 36 & 50.7 & 39 & 34.8 \\
\hline Professional only & 17 & 23.9 & 52 & 46.4 \\
\hline Missing & 18 & 25.4 & 21 & 18.8 \\
\hline \multicolumn{5}{|l|}{ ORIENTATION \& MANAGEMENT } \\
\hline \multicolumn{5}{|l|}{ Twinning model best to achieve HRH Program goals } \\
\hline No & 8 & 11.3 & 39 & 34.8 \\
\hline Yes & 41 & 57.7 & 28 & 25.0 \\
\hline Maybe & 17 & 23.9 & 45 & 40.2 \\
\hline Missing & 5 & 7.0 & 0 & 0.0 \\
\hline \multicolumn{5}{|l|}{ When understood twinning } \\
\hline Once I started working / still do not understand & 36 & 50.7 & 43 & 38.4 \\
\hline Before or during orientation & 28 & 39.4 & 69 & 61.6 \\
\hline Missing & 7 & 9.9 & 0 & 0.0 \\
\hline \multicolumn{5}{|l|}{ HRH Program goals are clear } \\
\hline Yes & 60 & 84.5 & 79 & 70.5 \\
\hline Missing & 11 & 15.5 & 33 & 29.5 \\
\hline \multicolumn{5}{|l|}{ Nature of any difficulty with twin } \\
\hline No difficulty & 39 & 54.9 & 59 & 52.7 \\
\hline Task related or clinical difficulty & 5 & 7.0 & 5 & 4.5 \\
\hline Time or availability difficulty & 8 & 11.3 & 20 & 17.9 \\
\hline Missing & 19 & 26.8 & 28 & 25.0 \\
\hline \multicolumn{5}{|l|}{$\begin{array}{l}\text { Cultural differences: \% of } 16 \text { items that were 'moderately' } \\
\text { or 'extremely' different }\end{array}$} \\
\hline Less than half moderate or extremely different & 18 & 25.4 & 9 & 8.0 \\
\hline More than half moderate or extremely different & 25 & 35.2 & 88 & 78.6 \\
\hline Missing & 28 & 39.4 & 15 & 13.4 \\
\hline
\end{tabular}


Table 1 Summary of covariates by faculty type (Continued)

\begin{tabular}{|c|c|c|c|c|}
\hline \multicolumn{5}{|c|}{ HRH Program promotes a culture of respect } \\
\hline Yes & 21 & 29.6 & 28 & 25.0 \\
\hline Most of the time & 15 & 21.1 & 52 & 46.4 \\
\hline Rarely & 7 & 9.9 & 17 & 15.2 \\
\hline Missing & 28 & 39.4 & 15 & 13.4 \\
\hline \multicolumn{5}{|c|}{ Senior leadership at work site support HRH Program } \\
\hline Agree or strongly agree & 40 & 56.3 & 45 & 40.2 \\
\hline Disagree or strongly disagree & 2 & 2.8 & 24 & 21.4 \\
\hline Neutral & 1 & 1.4 & 30 & 26.8 \\
\hline Missing & 28 & 39.4 & 13 & 11.6 \\
\hline Overall & 71 & 100 & 112 & 100 \\
\hline
\end{tabular}

at least one language, having a relationship that is both professional and social) and orientation or management factors (belief that HRH Program promotes a culture of respect) were associated with goal setting in bivariate analysis $(p<0.1)$ (Table 2). In multivariate analysis, significant prior teaching experience, feeling valued by one's twin and being within 5 years of age were associated with goal setting among USF $(p<0.1)$ (Table 2).

\section{Skills transfer}

Among those who responded to questions about skill transfer, half (52\%) of RF reported "very effective" skill transfer from their USF twin, whereas less than $10 \%$ of USF felt they transferred skills very effectively to their RF twin (Table 1). In bivariate analysis of RF, three factors were significantly associated with skill transfer (at $p<0.1$ ): participating in a nursing program outside of Kigali compared to the main university campus or working at multiple hospitals, being seven or more years post training and having excellent communication with twin in one or more languages (Table 2). Sharing a common language and being in a nursing program outside of Kigali remained significant in multivariate regression $(p<0.1)$ (Table 2). Among USF, sharing a professional and social relationship with their twin and believing that HRH Program promotes a culture of respect were associated with perceived skill transfer to a Rwandan twin in bivariate analysis $(p<0.1)$, though neither factor remained significant in multivariate analysis when satisfaction was in the model $(p<0.1)$ (Table 2).

\section{Satisfaction}

Among those who answered questions about satisfaction, $38 \%$ of RF and $28 \%$ of USF reported they were "very satisfied" with the twinning experience overall (Table 1). In bivariate analysis, factors associated with RF satisfaction were: being seven or more years post training, having a lot of experience with people from Western culture before engagement in the HRH Program and being a male RF twinned with a female USF versus same gender pairing $(p<0.1)$ (Table 2). Being seven years post training and male RF-female USF pairs remained significant in multivariate analysis $(p<0.1)$. Among USF in bivariate analysis, satisfaction was

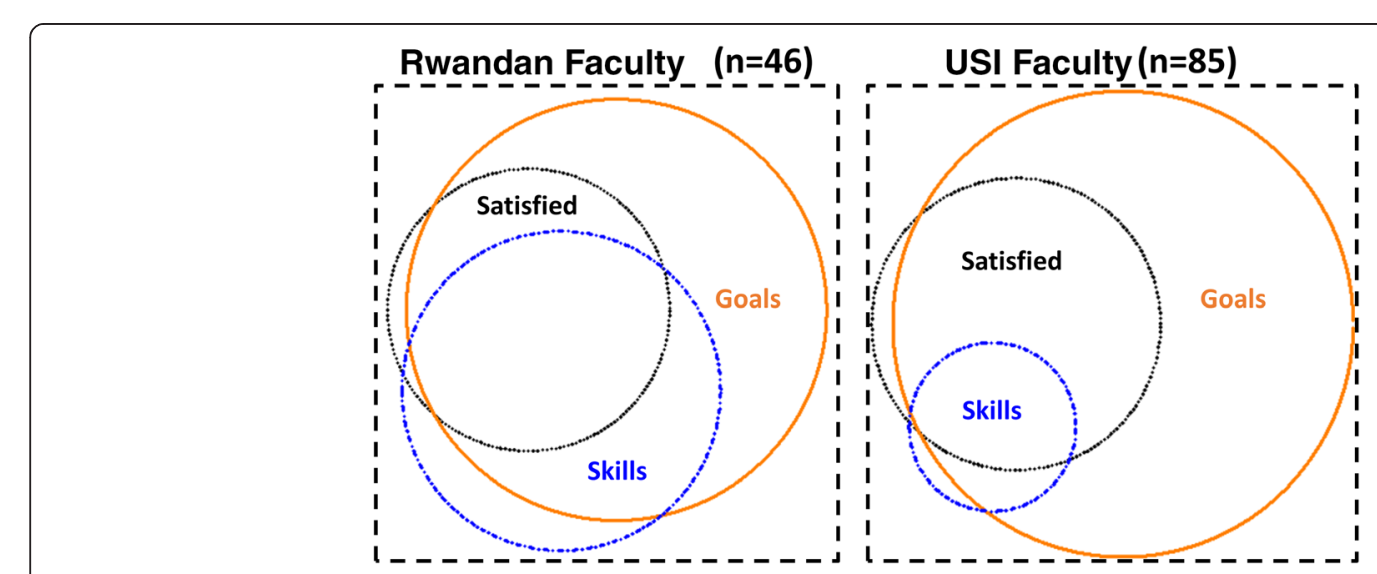

Fig. 2 Magnitude of outcome variables and their overlap, by USI faculty and Rwandan faculty. The box represents $100 \%$ of respondents who answered questions about all three outcomes. The size of the circle represents proportion reporting the outcome. The overlap in circles represents overlap in outcomes reported. 
predicted by a number of hiring or appointment factors (spent less than a month in low-resource countries at a time prior to engage in HRH Program, feel he/she is applying own talent and expertise, feel he/she is adequately mentoring others in educator, clinical, research, or administrator roles, and twin handles criticism well), twinning factors (have excellent communication in at least one language, share a professional and social relationship with twin) and orientation or management factors (belief that twinning model is the best way to achieve HRH Program goals, feel that HRH Program promotes a culture of respect, and the senior leadership at sites support the HRH Program) $(p<0.1)$ (Table 2). In multivariate analysis, having prior experiences of less than a month in low-resource countries and feeling the HRH Program promotes a culture of respect and work site senior leadership support the HRH Program remained significant $(p<0.1)$.

\section{Discussion}

In this survey, the link between satisfaction, spending time with HRH twin, goal setting and perceived skill transfer was very strong. The highest performing outcome was goal setting, with $89 \%$ of RF and $71 \%$ of USF reporting that they had set joint goals with their twin. While the proportion of faculty who were "very satisfied" was only $29 \%$ among USF and $38 \%$ among RF, most faculty (93 \% RF, $59 \%$ USF) expressed satisfaction to some extent. Central to the overall mission of the HRH Program, the lowest performing outcome was effective skill transfer; interestingly, this result was disparate between the RF and USF with $50 \%$ and $10 \%$ reporting effective skill transfer, respectively. It should be noted that all items were self-reported perceptions and all, particularly the effective skill transfer, could be based on cultural or historical expectations such that what RF or USF faculty believe to be effective skills transfer may vary. The findings of important factors that may improve RF and USF twinning experiences are described in more detail below.

\section{Recruitment and onboarding of US institution faculty}

We were surprised to find that USF with the most overseas experience were the least satisfied with the HRH Program. We assumed extensive prior work overseas would help the faculty set expectations and prepare for their engagement in HRH Program experience. However, the HRH Program uses a unique model with the USF working on a country-developed agenda under the leadership of Rwandans. We suspect this is different from other programs where the USF may have mostly held leadership positions and developed their own workplan. Indeed, previous studies have shown that satisfaction among working professionals in Western settings is closely linked with decision-making ability on the job [25]. As such, the expectations drawn from the USF's prior extensive overseas experience may not have been met during their work with the HRH Program.

We are certainly not suggesting that in future years the HRH Program should not recruit USF with previous international experience. However, we do think sufficient pre-Rwanda training, focusing on linking faculty's experiences to what can be expected from their time in Rwanda will be key for managing expectations and increasing overall satisfaction [26]. Further, the orientation should include clear information on the HRH Program and more details on work expectations, the twinning model, the health and education systems in Rwanda as well as cross-cultural matters to better prepare all USF for their engagement in HRH work.

\section{Recruitment and onboarding of Rwandan faculty}

Compared to most professional development programs that focus on new faculty [27], the HRH Program targets RF of various levels. In this survey, RF with more than seven years of experience reported the highest level of skill transfer and were more likely to be satisfied by the twinning experience. In other studies in the United States, senior faculty showed interest in professional development programs such as mentorship [28, 29]; while the Rwandan context has marked differences, we believe that similarly, more experienced faculty will value the opportunity to learn from USF and will be better able to articulate concrete learning goals for the twinning process.

We hypothesize the finding that less experienced Rwandan colleagues were less satisfied and perceived less effective skill transfer may also reflect two challenges that were observed in studies from the United States: 1) low motivation because they are unclear how the gain of skills through the HRH Program will tangibly lead to personal and professional promotion [29] and 2) lack of time to participate. In the future, if these faculty are selected as targets for the HRH Program, then we recommend allocating dedicated time to participate and to award their participation during faculty performance appraisal and with credit for continuing medical education [27].

\section{Twinning matters}

The twinning model creates a framework in which skills are transferred primarily to the RF, and thus how twins are paired is important to the success of the program. The best way to match twins is not yet established. Some programs assign pairs [27] and other programs allowing pairs to self-identify [28, 30]. For the HRH Program, each department has generally assigned pairs, weighing the training needs of the RF with the skills of the USF. 
Table 2 Factors associated with operational outcomes in bivariate and multivariate analysis, by faculty type

\begin{tabular}{|c|c|c|c|c|c|c|}
\hline & \multicolumn{2}{|l|}{ Goals } & \multicolumn{2}{|l|}{ Skills } & \multicolumn{2}{|l|}{ Satisfaction } \\
\hline & Rwandan Faculty & USI Faculty & Rwandan Faculty & USI Faculty & Rwandan Faculty & USI Faculty \\
\hline \multicolumn{7}{|l|}{ OUTCOMES } \\
\hline \multicolumn{7}{|c|}{ Very effective skill transfer (USF-to-RF) } \\
\hline No & & & & & - & - - \\
\hline Yes & & & & & ++ & ++++ \\
\hline \multicolumn{7}{|c|}{ Set joint goals with twin } \\
\hline No & & & & & & ++ \\
\hline Yes & & & & & & - \\
\hline \multicolumn{7}{|c|}{ Very satisfied with twinning experience overall } \\
\hline No & & - & - - & -— & & \\
\hline Yes & & ++ & ++++ & ++++ & & \\
\hline \multicolumn{7}{|c|}{ Number of hours per week spent working with twin } \\
\hline $0-9$ hours & & - & & & -— & - - \\
\hline 10-19 hours & & & & & ++ & \\
\hline $20+$ hours & & ++++ & & & ++++ & ++++ \\
\hline
\end{tabular}

HIRING AND APPOINTMENTS

Primary work site

CMHS, multiple

CHUB, CHUK, KFH, RMH, Muhima

Nursing outside Kigali

Years since training

$7+$ years

4-6 years

$1-3$ years

Completed prior to HRH, not yet completed

Previous teaching experience

Moderate-little teaching

Significant teaching

Time spent in resource limited countries before HRH Program

Short trips $<1$ month, none

$$
++
$$

Medium trips $<6$ months

Long trips $>6$ months

Applied talent and expertise

Agree, strongly agree

Do not agree

Experience working with people from Western culture before HRH Program

A lot

\section{Moderate}

None or minimal

Twin values ALL of the following: my expertise, my opinion, our department hierarchy, my professional interests or goals 
Table 2 Factors associated with operational outcomes in bivariate and multivariate analysis, by faculty type (Continued)

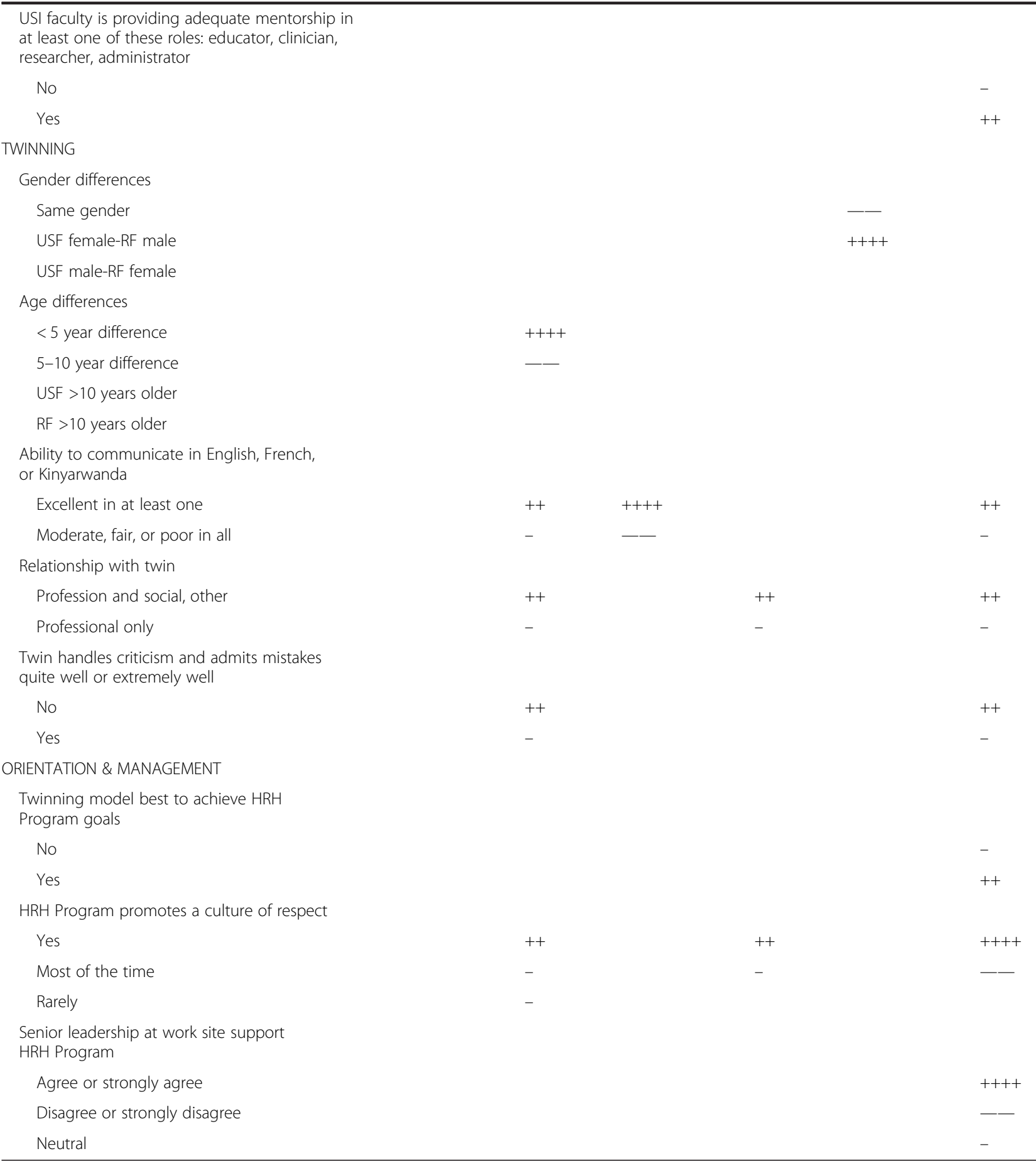

Code: - indicates significantly lower on univariable, _- indicates significantly lower in multivariable; ++ indicates significantly higher on univariable ++++ indicates significantly higher in multivariable

However, we note from these survey results that the twinning process should also consider the age, gender and language competencies of the twins. We found that twins of approximately the same age more likely to set goals together, which is consistent with other programs that found large age differences may promote a hierarchy that prevents effective learning in junior faculty [29]. Unexpectedly, male RF reported more satisfaction when matched with female USF. This is contrary to other studies that recommend same-gender pairs [28] and should be explored more in future surveys. Inability to communicate in the same language was linked to lower likelihood of setting goals and 
lower satisfaction in USF and lower likelihood to report effective skill transfer in RF. Since many USF are not fluent in French and very few speak Kinyarwanda, ideally the initiation of RF should make them fluent in English, the official language of Rwanda since 2011. We will also encourage USF to take French lessons prior the joining the twinning program, and as time and resources allow, provide some French training within the HRH Program.

\section{The role of cross-cultural training and exchanges}

In addition to professional relationships with the twin, it turns out that social relationships are also important. Social connections would allow USF to feel more integrated, to gain a deeper understanding of their twin and to understand some of the cultural content surrounding medical practice [31-33]. We also believe that other factors identified in this study could be linked to cross-cultural misunderstandings. RF with more experience working with someone from Western cultures were more likely to be satisfied with the HRH Program. Further, USF who felt that their expertise or opinions were not valued were less likely to set goals and USF who felt their twin did not handle criticism well were less likely to set goals and be satisfied with the HRH Program. We hypothesize that perception of how others value expertise or handle criticism may be influenced by cultural lenses, and that the perception of these characteristics could be improved with cross-cultural sensitization on Rwandan-Western cultural norms. In future years, the HRH Program plans to integrate cross-cultural training into RF and USF orientations, and to nurture social relationships and cross-cultural exchanges through sponsored non-work activities.

\section{Modeling effective programs}

Nursing sites outside of Kigali were linked to more effective skill transfer, and the reasons are not entirely clear. One possible explanation is that the nursing programs outside Kigali are located in semi-urban areas where interactions among nursing faculty more easily blend social and professional life compared to faculty located in Kigali or assigned to multiple teaching sites. Further, RF at these nursing sites are often enrolled in formal training and degree upgrading programs, so they may benefit from their twin both directly through $\mathrm{HRH}$ Program activities and through informal support for their work in these training programs. We recommend further investigation to understand why skill transfer at nursing sites outside of Kigali was perceived to be so effective, which might improve skill transfer at teaching hospitals and the university located in Kigali.

\section{HRH Program buy-in at the institutional level}

To make twinning more successful and gratifying for USF and RF, HRH Program leadership both at central and teaching sites should be actively involved with creating collaborative work environments. USF who felt supported by site leadership were more satisfied with the twinning process, and we believe that this is linked to receiving support needed to achieve twinning goals. The fact that some site leaders were not heavily engaged in the initial $\mathrm{HRH}$ years is a function of the limited human resources at the health institution leadership level, such that they were unable to provide necessary support or resources due to competing institutional demands. To address this, the $\mathrm{HRH}$ Program has started a series of meetings with UR-CMHS and department leadership to discuss challenges and provide solutions to overcome potential barriers for effective twinning and skills transfer at site.

\section{Limitations}

Several limitations should be considered in the interpretation of these results. The present study targeted all faculty who participated in the program in the period between $\mathrm{Au}$ gust 2012 and May 2013; however, it is possible that we were unable to invite some faculty as a result of our inability to make contact with them such as having an incorrect email address. Further, RF participation in the survey was lower compared with USF participation. This may have been due the fact that both clinical and classroom teaching work kept them busy such that finding time to participate in the study was difficult. In addition the use of an online survey may have reduced the number of RF responding due to limited previous exposure to online surveys and poor internet access. The questionnaire was long, which may have increased missing rates for later questions. The survey may have had recall biases, particularly for faculty who had not been in the HRH Program for over a year. Finally, although respondents were assured that no directly identifiable information would be collected, respondents who had unique demographic or job profiles in the program may have been less forthcoming with criticism if they felt responses could not be anonymized. However, despite these limitations, we believe these results are informative, both because of the uniqueness of the twinning model in the HRH Program and because this is the first formal assessment of the satisfaction and perceived effectiveness of this program. We intend to follow-up this assessment with $\mathrm{RF}$ and USF interviews to corroborate these findings and to better understand some of the associations observed.

\section{Conclusions}

Overall, the HRH Program has been useful for improving the skills of the health workforce in Rwanda. However, central to the HRH Program is the twinning model, specifically goal setting and skills transfer between twins, and satisfaction with the twinning model is important for the HRH Program success. The findings of this study helped the HRH team identify key areas to improve the 
twinning experience. For example, following information gained from this survey, the program has improved preorientation of USF and RF, it is increasing its staff support to assist cross-cultural training and social activities, and studying best practices of the nursing sites. Other factors such as age and gender in the matching process need more investigation, but the HRH team is willing to advise on considering these factors in the matching of RF-USF twins if supported by the evidence.

To ensure continuous improvement of twinning model, mechanisms to collect feedback on the twinning process will be reinforced to expand best practice and manage reported challenges. This will both optimize the HRH Program in Rwanda and provide information that can contribute to the development of health workforces in other low-income countries through implementation of effective models.

\section{Additional file}

Additional file 1: Univariate and multivariate associations between all covariates and outcomes, by faculty type. (DOCX $77 \mathrm{~kb}$ )

\section{Competing interests}

The authors declare that they have no competing interests.

\section{Authors' contributions}

The evaluation tool was developed by a large, diverse group that included all coauthors and others with leadership by MW. This analysis plan was conceived by EN, GU, EU, JM. BHG, DRT, and EU provided analysis support. EN and GU led writing of this manuscript with extensive inputs from BHG. DT provided extensive support on the development of methodology and analysis. AB designed, oversaw development and implementation of HRH Program; she initiated this study and provided guidance on writing this paper. All authors provided input, and reviewed and approved the final version.

\section{Acknowledgements}

We acknowledge the host of individuals who conceived and championed the Human Resources for Health Program in Rwanda. A special thanks to the following individuals who helped to design the evaluation tool: Phil Cotton, Cari Davis, Ida Kankindi, Angele Musabyimana, Chris Paletta, April Ricotta, Stephen Rulisa, J Paul Semasaka, and Philomene Uwimana.

\section{Author details}

'Human Resources for Health, Ministry of Health, P.O. Box 84, Kigali, Rwanda. ${ }^{2}$ College of Medicine and Health Sciences, University of Rwanda, P.O. Box 3286, Kigali, Rwanda. ${ }^{3}$ Department of Global Health and Social Medicine, Harvard Medical School, 641 Huntington Avenue, Boston, MA 02115, USA. ${ }^{4}$ Ministry of Health, P.O. Box. 84, Kigali, Rwanda. ${ }^{5}$ Geisel School of Medicine, 1 Rope Ferry Road, Hanover, NH 03755, USA.

Received: 18 June 2015 Accepted: 12 January 2016

Published online: 28 January 2016

\section{References}

1. Dussault G, Dubois CA. Human resources for health policies: a critical component in health policies. Hum Resour Health. 2003;1:1.

2. World health report. Working together for health. Geneva: World Health Organization 2006. [www.who.int/whr/2006/en/index.html]. Accessed 15 Apr 2015.

3. Crisp N, Gawanas B, Sharp I. Training the health workforce: scaling up, saving lives. Lancet. 2008;371:9613.

4. Tankwanchi ABS, Özden C, Vermund SH. Physician emigration from SubSaharan Africa to the United States: analysis of the 2011 AMA physician master file. PLoS Med. 2013;10:12.
5. Salsberg E, Grover A. Physician workforce shortages: implications and issues for Academic Health Centers and policymakers. Acad Med. 2006;81:9.

6. Gupta N, Diallo K, Zurb P. Poz, MRD: assessing human resources for health: what can we can be learned from labour surveys. Hum Resour Health. 2003;1:5.

7. Machey TR, Liang BA. Rebalancing brain drain: exploring resource reallocation to address health worker migration and promote global health. Lancet. 2012;107:1.

8. Hongoro C, McPake B. How to bridge the gap in human resources for health. Lancet. 2004;364:9443.

9. Anyangwe SCE, Mtonga C. Inequities in the Global Health Workforce: the greatest impediment to health in Sub-Saharan Africa. Int J Environ Res Public Health. 2007:4:2.

10. Mullan F, Frehywot S, Omaswa F, Buch E, Chen C, Greysen SR, et al. Medical schools in sub-Saharan Africa. Lancet. 2011:377:9771.

11. World Health Statistics. Workforce, Infrastructure, essential medicines. Geneva: World Health Organization. 2009. [http://www.who.int/whosis/ whostat/EN_WHS09_Table6.pdf]. Accessed 15 Apr 2015.

12. McCourt W, Awases M. Addressing the human resources crisis: a case study of the Namibian health service. Hum Resour Health. 2007;5:1.

13. World Health Organization. World Health Statistics 2010. Geneva: World Health Organization; 2010.

14. United Nations. The Millennium Development Goals Report 2015. New York: United Nations; 2015.

15. Palmer D. Tackling Malawi's human resources crisis. Reprod Health Matters. 2006;14:27.

16. Kaaya EE, Macfarlane SB, Mkony AC, Lyamuya FE, Loeser F, Freeman $\mathrm{P}$, et al. Educating enough competent health professionals: advancing educational innovation at Muhimbili University of Health and Allied Sciences, Tanzania. PLoS Med. 2012;9:8.

17. Drobac P, Naughton B. Health equity in Rwanda: the New Rwanda, twenty years later. Harvard Int Rev. 2014;35:4.

18. World Health Organization. World Health Statistics 2014. Geneva: World Health Organization; 2014

19. Farmer $P E$, Nutt $C T$, Wagner $C M$, Sekabaraga $C$, Nuthulaganti T, Weigel JL, et al. Reduced premature mortality in Rwanda: lessons from success. BMJ. 2013;346:5534

20. Human Resources for Health Program. Strategic plan 2011-2016. Kigali: Ministry of Health of the Republic of Rwanda; 2011. (http://www.brown. edu/academics/medical/bright/sites/brown.edu.academics.medical.bright/ files/uploads/MOH\%20Rwanda\%20HRH\%20Strategic\%20Plan\%202011\%20\%202016.pdf). Accessed 15 Apr 2015.

21. Binagwaho A, Kyamanywa P, Farmer PE, Nuthulaganti T, Umubyeyi B, Nyemazi JP, et al. The Human Resources for Health Program in Rwanda - a new partnership. N Engl J Med. 2013;369:21.

22. Rwanda Human Resources for Health Program 2011-2019: funding proposal. Kigali: Ministry of Health of the Republic of Rwanda; 2011. (https://medicine. yale.edu/intmed/global/sites/Rwanda\%20HRH\%20Proposal\%20FINAL_ 129987_284_7289.pdf). Accessed 18 Jan 2016.

23. Smith $\mathrm{C}$. The role of health links in international development: the need for greater evidence? Trop Doct. 2012;42:65-6.

24. Ritman $D$, Zegeye $H$. The value of health partnerships: a practical approach to evidence. Trop Doct. 2012;42:241-2.

25. Laschinger SHK, Finegan J, Shamian J. The impact of workplace empowerment, organization trust on staff nurses' satisfaction and organizational commitment. Health Care Manage Rev. 2001;26:3.

26. Balandin S, Lincon M, Sen R, Wilkins DP, Trembath D. Twelve tips: Twelve tips for effective international clinical placements. Med Teach. 2007;29:9-10

27. Thorndyke LE, Gusic ME, George JG, Quillen DA, Milner RJ. Empowering junior faculty: Penn State's: faculty development and mentoring program. Acad Med. 2006;81:7.

28. Binkley PF, Brod HC. The mentorship gap in an academic medical center: a needs assessment for structured mentorship programs. Am J Med. 2013;126:11

29. Carr PL, Pololi L, Knight S, Conrad P. Collaboration in academic medicine: reflections on gender and advancement. Acad Med. 2009;84:10.

30. Varkey P, Jatoi A, Williams A, Mayer A, Ko M, Files J, et al. The positive impact of a facilitated peer mentoring program on academic skills of women faculty. BMC Med Educ. 2012;12:14. 
31. Robert JC, Steven PD. Caring for the whole person: guidelines for advancing undergraduate mentorship. Coll Stud J. 2007;41:4.

32. Schrubbe KF. Mentorship: a critical component for professional growth and academic success. J Dent Educ. 2004:68:3.

33. Straus SE, Johnson MO, Marquez C, Feldman MD. Characteristics of successful and failed mentoring relationships: a qualitative study across two academic health centers. Acad Med. 2013;88:1.

Submit your next manuscript to BioMed Central and we will help you at every step:

- We accept pre-submission inquiries

- Our selector tool helps you to find the most relevant journal

- We provide round the clock customer support

- Convenient online submission

- Thorough peer review

- Inclusion in PubMed and all major indexing services

- Maximum visibility for your research

Submit your manuscript at www.biomedcentral.com/submit 